\title{
Microbiota therapeutic RBX2660 shows promising results in patients with recurrent Clostridioides difficile infection
}

\author{
Suryang Kwak \\ JooHee Choi \\ Tiffany Hink \\ Kimberly A. Reske \\ Kenneth Blount \\ Courtney Jones \\ Margaret H. Bost \\ Xiaoqing Sun
}

Carey-Ann D. Burnham

Erik R. Dubberke

Gautam Dantas for the CDC Prevention Epicenter Program

\section{Video Byte}

Keywords: Microbiome, microbiota-based therapy, placebo, resistome, Clostridioides difficile infection, antibiotic-resistant organisms, ARO, ARG, antibiotic resistance gene, rCDI, fecal microbiota transplantation, FMT, intestinal dysbiosis, microbiota restoration therapy, antibiotics, abundance, alpha diversity, pathogen, RBX2660, E. coli

Posted Date: November 12th, 2020

DOI: https://doi.org/10.21203/rs.3.rs-106668/v1

License: (c) (i) This work is licensed under a Creative Commons Attribution 4.0 International License. Read Full License 


\section{Abstract}

Fecal microbiota transplantation is a promising procedure for preventing recurrent Clostridioides difficile infection ( $\mathrm{rCDI}$ ), which is the most frequently identified healthcare-associated infection in the US. Unfortunately, the effects of microbiota transplantations on the microbiome and resistome of rCDI patients have not been examined in detail. To address that gap, researchers recently conducted a randomized, double-blind, placebo-controlled clinical trial of the investigational microbiota therapeutic RBX2660 for patients with rCDI. Over the first 7 days after treatment, all patients showed significant recovery of gut microbiome architecture and a decreased abundance of antibiotic-resistance genes. However, patients receiving RBX2660 showed more significant and longer-lasting microbiome and resistome shifts toward a balanced configuration than those receiving the placebo. Whole genome sequencing revealed the eradication of antibiotic-resistant organisms in patients receiving RBX2660, while also underscoring the challenges of potential transmission of common antibiotic-resistant organisms, emphasizing the need for careful screening of microbiota therapeutics. Overall, RBX2660 showed the potential to restore microbiome balance and reduce intestinal antibiotic-resistant organisms, making it a promising therapeutic for the recovery of the microbiome after antibiotics, as well as prevention of rCDI. 